\title{
Assessment of Lumbar Spine Kinematics by Posterior-to-Anterior Mobilization
}

\author{
Kang O Oh ${ }^{a}$ and Sang-Yeol Lee ${ }^{b^{*} \oplus \mathbb{D}}$

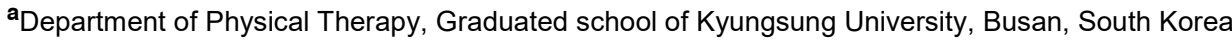 \\ bepartment of Physical Therapy, Kyungsung University, Busan, South Korea
}

Objective: Studies confirming the lumber spine kinematics of direct or indirect segmental mobility under the application of joint mobilization, which induces passive force on the spine, are insufficient.Therefore, this study aims to obtain the underlying clinical data by identifying direct or indirect segmental mobility produced by Maitland's PA mobilization technique.

Design: Randomized controlled trial design.

Methods: Thirty subjects with no back pain participated in this study. X-ray testing equipment (SIG-40-525, Ecoray Inc., Korea) was used to verify the segmented movement of their lumbar. Joint mobilization was performed by physiotherapists with more than 10 years of experience in prescription therapy, and radiography was performed once without PA joint mobilization and once without the mobilization for comparing the lumbar vertebrae before and after the mobilization. The radiographs taken were analyzed using the picture archiving and communication system (PACS) program to measure the spinal displacement, intervertebral height, intervertebral angle, and lumbar lordosis angle.

Results: Significant differences were observed in the lumbar displacement, intervertebral angle, and lumbar lordosis angle in all lumbar vertebrae before and after the mobilization. The intervertebral height indicated significant differences in all ventral vertebrae and only in L3-L4 and L4-L5 in dorsal vertebrae.

Conclusions: This study suggests that the segmental mobility produced through indirect approaches plays an important role in inducing therapeutic effects in patients with back pain.

Key Words: Lumbar mobilization, Lumbar segmental mobility, Manual therapy, Kinematics

\section{Introduction}

Since its introduction in the 18th century, spine joint mobilization has gained increasing popularity as the most commonly used passive movement technique to treat and manage musculoskeletal disorders in the spinal region [1-3]. This technique applies large and slow rhythmic movements, including passive sliding, distraction, rolling, spinning, and compression to the joint surface, within the range of motion of the vertebrae [4-6].

A typical Maitland technique of joint mobilization is based on the concept of a brick wall [7], in which the strength of joint mobilization is divided into five levels: Grades I-IV are used for joint mobilization and Grade $\mathrm{V}$ is used for joint manipulation [8]. In Grades I-II, the therapist performs the first pre-resistance movement of the joint, which corresponds to the point at which the connective tissue begins to impose significant resistance to deformation. Grades III-IV indicate an action that occurs from the point of initial resistance to that of maximum resistance, which determines the end range of the accessory motion [9-10]. Maitland recommended the use of posteroanterior (PA) joint mobilization in the prone position on patients with lower back pain [11], This technique improves the relaxation and mobility of

Received: Dec 7, 2021 Revised: Dec 14, 2021 Accepted: Dec 15, 2021

Corresponding author: Sang-Yeol Lee (ORCID https://orcid.org/0000-0003-4428-9101)

Graduated school of Kyungsung University, Kyungsung University, 309, Suyeong-ro, Nam-gu, Busan, Republic of Korea

Tel: +82-51-663-4873 Fax: +82-51-663-5059 E-mail: sjslh@ks.ac.kr

This is an Open-Access article distributed under the terms of the Creative Commons Attribution Non-Commercial License (http://creativecommons.org/licenses/ by-nc/4.0) which permits unrestricted non-commercial use, distribution, and reproduction in any medium, provided the original work is properly cited.

Copyright $(2021$ Korean Academy of Physical Therapy Rehabilitation Science 
the joint-peripheral soft tissue due to limited mobility [12]. The normal movement of the damaged joints prevents the deterioration of the symptoms, and the sense of inherent proprioception through joint movements stimulates the normal nerve function to inhibit pain and muscle imbalance [6].

To obtain positive clinical effects of passive joint mobilization, the spine should be subjected to a sufficient force that produces segmental motion [5]. An inadequate force applied during PA joint mobilization can not only result in negative clinical effects but also cause excessive stress in the anatomical structure of the spine [13]. When defining segmental mobility under the force applied to the spine, the distance between the start point, which indicates the position of the spine before segmentation, and end point, which indicates its position after segmentation, is calculated [14]. A recent study showed that, under the application of passive PA forces, the segmental mobility of the adjacent vertebrae that are not subjected to any direct force is likely $3^{\circ}-4^{\circ}$ [15]. In addition, the contact between the L3 inferior and L4 superior articular processes causes the segmental mobility of L4 [15]. Therefore, in this study, in addition to the segmental mobility under the application of passive force, the rotation of the sagittal plane and the mobility of other segments were also observed[15].

However, the research identifying the form and magnitude of direct or indirect segmental mobility that applies passive force to the spine remains insufficient at home and abroad. Therefore, this study aims to obtain the underlying clinical data by identifying direct or indirect segmental mobility produced by Maitland's PA mobilization technique.

\section{Methods}

\section{Study population}

In this study, 30 adult men and women in their $20 \mathrm{~s}$ and 40s (male, 16 and female, 14) were randomly selected from OOO Hospital in Busan for a period of two months from July to August 2021 (Table 1). Before the experiment, which was approved by the Institutional Review Board of Kyungsung University, a consent form was distributed to all subjects regarding their participation in the experiment; the purpose of the
Table 1. General characteristics of subjects (Means \pm SD)

$(\mathrm{n}=30)$

\begin{tabular}{lll}
\hline & Male $(\mathbf{n}=\mathbf{1 6})$ & Female $(\mathbf{n}=14)$ \\
\hline Age $(\mathrm{y})$ & $33.31 \pm 4.52$ & $28.07 \pm 2.81$ \\
Body mass $(\mathrm{kg})$ & $74.94 \pm 7.18$ & $56.30 \pm 5.66$ \\
Body height $(\mathrm{cm})$ & $175.48 \pm 6.05$ & $160.17 \pm 3.23$ \\
BMI $\left(\mathrm{kg} / \mathrm{m}^{3}\right)$ & $24.32 \pm 2.05$ & $22.01 \pm 1.89$ \\
\hline
\end{tabular}

study was explained to all of them and voluntary consent was obtained from all. The subjects who demonstrated any of the following characteristics were excluded from the experiment:

- A person with more than three days of back pain

- A person diagnosed with lumbar disc and stenosis

- A person with musculoskeletal pain

\section{Study protocol}

The segmental mobility of the spine was measured using X-ray inspection equipment (SIG-40-525, Ecoray Inc., Korea). All subjects were positioned in the equipment, which was set up such that all lumbar bones were visible within the image plane. The subjects were asked to lie down on a hard bed in the prone position with their arms stretched next to the bed. The experimenter stood on the right side of the subject and performed PA joint mobilization for L3 segmentation using the pisiform of their lower hand with the assistance of the other hand. At this time, the subject's abdomen was supported by a small pillow placed underneath, and submaximum forces were applied to the spine. These forces belong to Grade IV and reach the maximum resistance point, which is the end range till which the joint can move without pain. Joint mobilization was performed by a physiotherapist with more than 10 years of experience in manual therapy. For a comparison of L3 before and after the mobilization, one radiograph was taken without PA joint mobilization and one with PA mobilization. The radiographs taken were analyzed using the picture archiving and communication system (PACS) program to measure the spinal displacement, intervertebral height, intervertebral angle, and lumbar lordosis angle. 


\section{Measurement}

\section{Lumbar displacement}

The PACS program (HI Pacs 4.0version, Medicore CO LTD., Korea) was used to determine the lumbar displacement before and after PA joint mobilization. Diagonal lines were drawn from each corner of the lumbar vertebral body to determine the lumbar center, designated as the starting point, and the highest point of the PACS program was selected as the end point to measure the degree of lumbar displacement (Figure 1-A).

\section{Intervertebral height}

The intervertebral height was determined as the distance from the horizontal line drawn on the bottom of L3 and that drawn on the top of L4. The lumbar vertebrae toward the end were defined as dorsal, whereas those in the front were defined as ventral (Figure 1-B).

\section{Intervertebral angle}

The angle between the horizontal line drawn on the lower surface of L3 and that drawn on the upper surface of L4 was measured as the intervertebral angle (Figure 1-C).

\section{Lumbar lordosis angle}

The angle between the horizontal line drawn on the top surface of L1 and that drawn on the top surface of S1 was measured as the lumbar lordosis angle (Figure 1-D).

\section{Statistical analysis}

The collected data were statistically analyzed conducted using the SPSS Ver. 25.0 (SPSS Inc., USA) statistical program. A paired t-test was conducted to compare the mean displacement, intervertebral height, intervertebral angle, and lumbar lordosis angle of the joint before and after the joint mobilization. In addition, the intraclass correlation coefficients (ICC3.1) were evaluated to validate the reliability of the measurement method. The value of these coefficient ranged $0.95-0.99$, indicating significantly high reliability of the method.

\section{Results}

PA mobilization was directly applied to L3 to confirm how joint mobilization affects the segmental mobility of the lumbar, and all movements of all lumbar vertebrae, including L3, were observed. At this time, Grade IV force was applied to the lumbar spine.

\section{Lumbar displacement}

The application of PA mobilization to L3 yielded significant differences in spinal displacement in all five lumbar vertebrae $(\mathrm{p}<0.000)$ (Table 2$)$. The displacement in L3 showed the maximum difference (from 24.51 \pm $2.25 \mathrm{~cm}$ to $27.73 \pm 2.1 \mathrm{~cm}$ ), whereas that in L5 showed the least difference (from $24.35 \pm 1.73 \mathrm{~cm}$ to $26.93 \pm$ $1.80 \mathrm{~cm}$ ).

\section{Intervertebral height}

In the dorsal vertebrae, the intervertebral height showed significant differences in L3-L4 $(\mathrm{p}<0.025)$ and L4-L5 $(\mathrm{p}<0.005)$. However, there was no difference in L1-L2 $(\mathrm{p}<0.139)$ and L2-L3 $(\mathrm{p}<0.835)$ (Table 3). In contrast, all ventral vertebrae, L1-L2, L2 -L3, L3-L4, and L4-L5 ( $\mathrm{p}<0.000)$, showed significant differences in the intervertebral height (Table 3 ).

\section{Intervertebral angle}

The intervertebral angle showed significant differences in all five vertebrae, L1-L2, L2-L3, L3-L4, and L4L5 $(p<0.000)$ (Table 4). Here, the L4-L5 angle showed the maximum difference (from $10.01 \pm 3.28^{\circ}$ to 13.35 $\pm 2.93^{\circ}$ ), whereas the L1-L2 angle showed the least difference (from $6.21 \pm 2.27^{\circ}$ to $8.38 \pm 1.97^{\circ}$ ).

\section{Lumbar lordosis angle}

The lordosis angle showed significant difference from $39.96 \pm 8.57^{\circ}$ to $52.52 \pm 8.06^{\circ}(\mathrm{p}<0.000)$ (Table 5). 

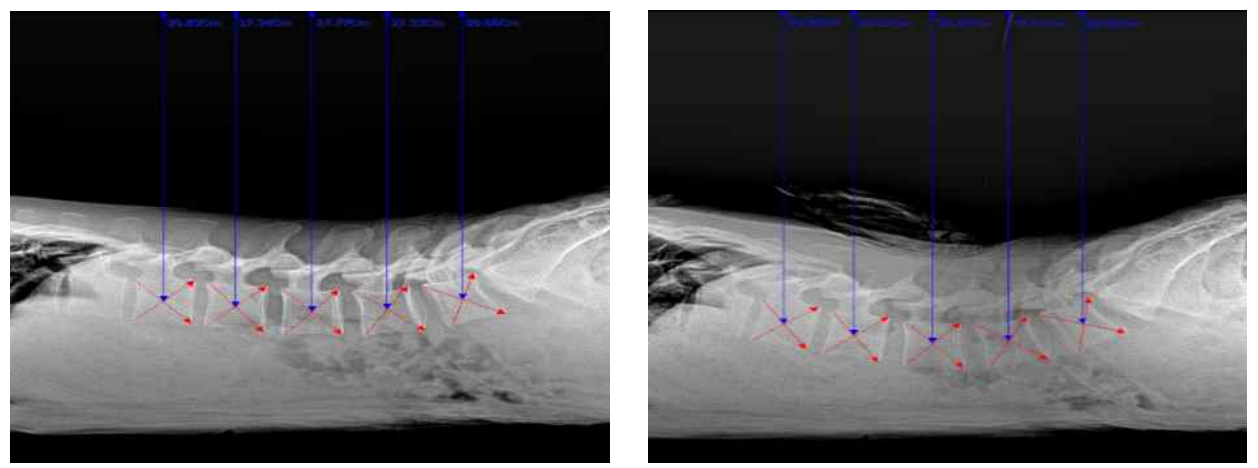

(A) Displacement
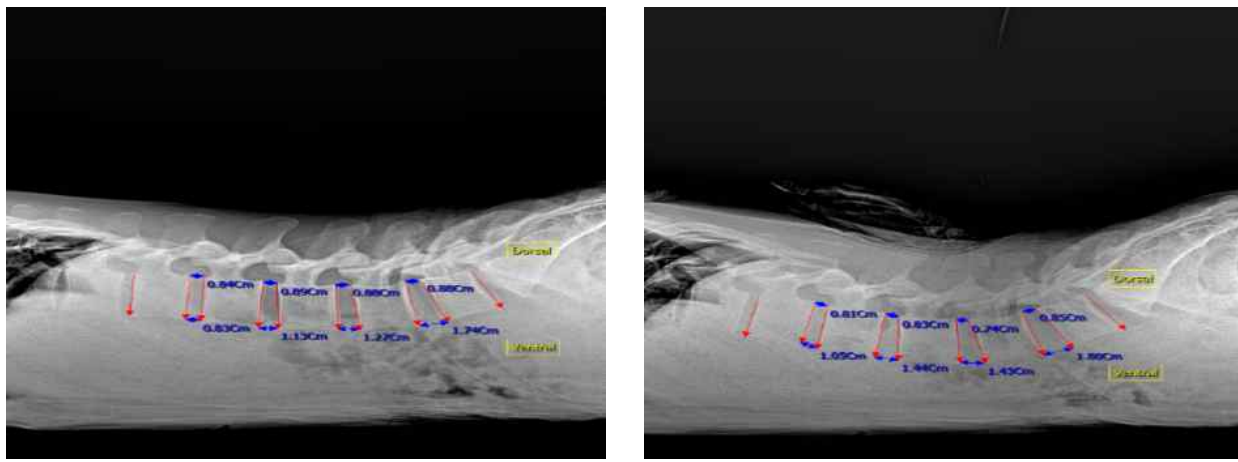

(B) Intervertebral Height
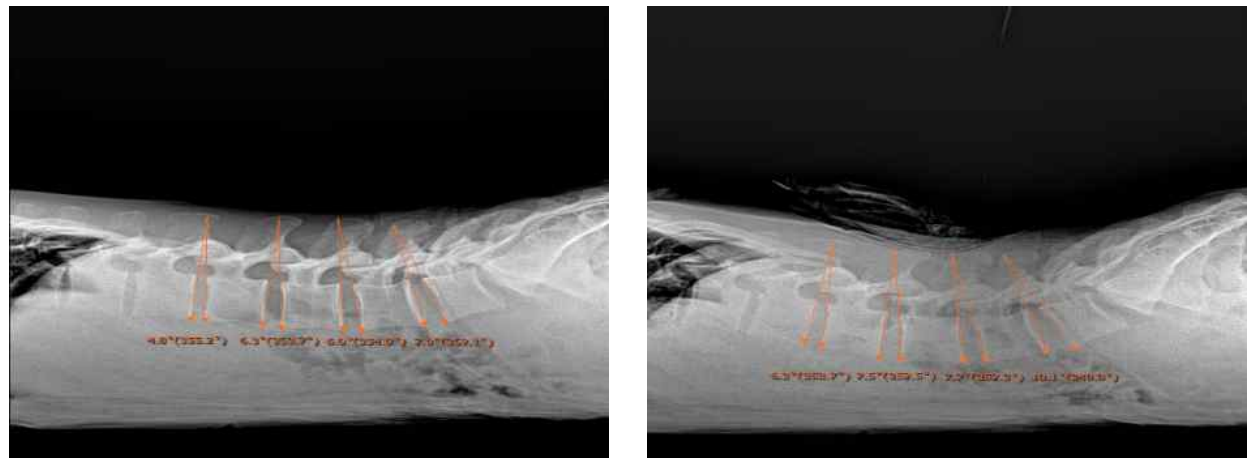

(C) Intervertebral Angle
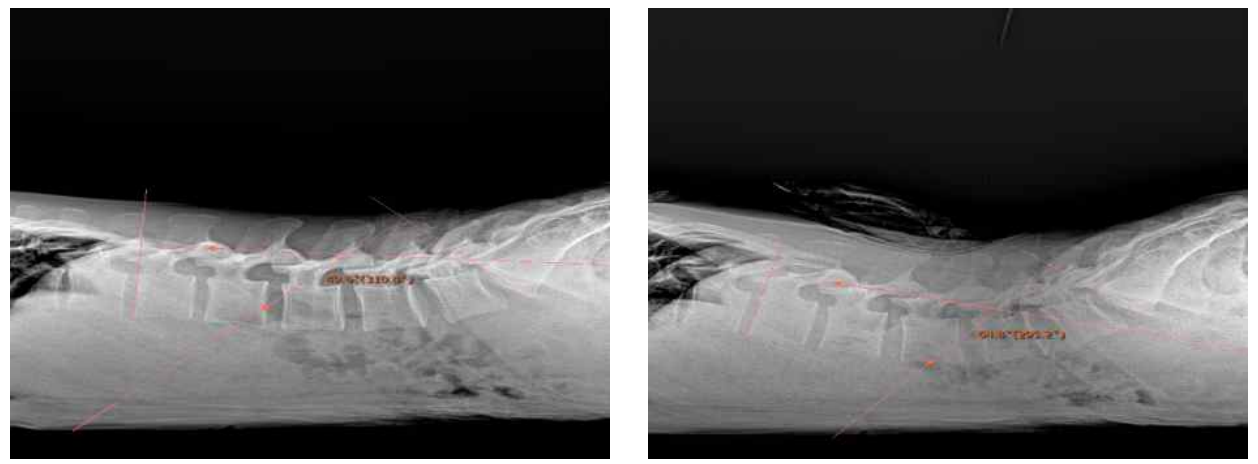

(D) Lumbar Lordosis Angle

Figure 1. Comparison of the L3 segment before and after joint mobilization.

(Left) resting position and (Right) mobilization from the end range 
Table 2. Comparison of lumbar displacement according to mobilization (Unit: $\mathrm{cm}$ )

\begin{tabular}{lllll}
\hline & Pre-test & Post-test & t & p \\
\cline { 2 - 3 } & Mean \pm SD & Mean \pm SD & -27.34 & $0.000^{*}$ \\
\hline Lumbar 1 & $23.09 \pm 2.36$ & $25.71 \pm 2.15$ & -28.57 & $0.000^{*}$ \\
Lumbar 2 & $23.81 \pm 2.30$ & $26.84 \pm 2.14$ & -26.49 & $0.000^{*}$ \\
Lumbar 3 & $24.51 \pm 2.25$ & $27.73 \pm 2.10$ & -24.53 & $0.000^{*}$ \\
Lumbar 4 & $24.77 \pm 2.08$ & $27.82 \pm 1.98$ & -22.28 & $0.000^{*}$ \\
Lumbar 5 & $24.35 \pm 1.73$ & $26.93 \pm 1.80$ & & \\
\hline
\end{tabular}

$* \mathrm{p}<0.05$, Mean \pm SD

Table 3. Comparison of intervertebral height according to mobilization (Unit: $\mathrm{cm}$ )

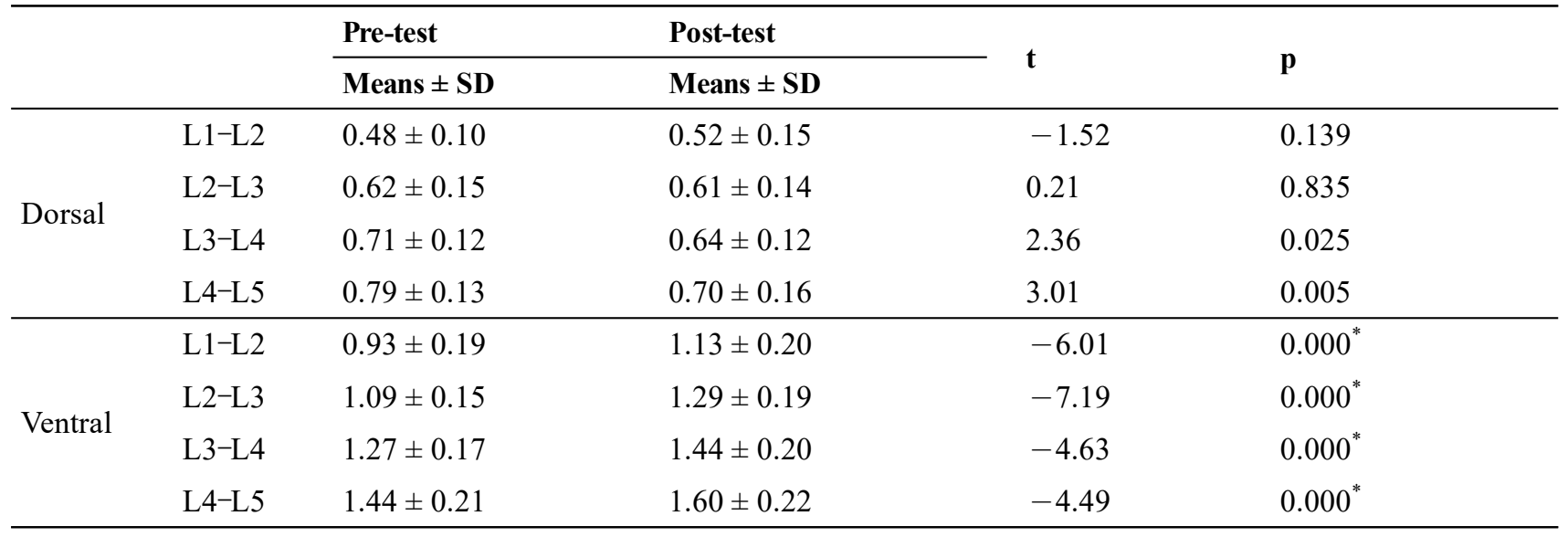

$* \mathrm{p}<0.05, \mathrm{Mean} \pm \mathrm{SD}$

Table 4. Comparison of intervertebral angle according to mobilization(Unit: ${ }^{\circ}$ )

\begin{tabular}{lllll}
\hline & Pre-test & Post-test & t & p \\
\cline { 2 - 4 } & Means \pm SD & Means \pm SD & -5.79 & $0.000^{*}$ \\
\hline L1-L2 angle & $6.21 \pm 2.27$ & $8.38 \pm 1.97$ & -11.22 & $0.000^{*}$ \\
L2-L3 angle & $6.64 \pm 1.77$ & $9.86 \pm 1.81$ & -6.53 & $0.000^{*}$ \\
L3-L4 angle & $8.23 \pm 2.23$ & $11.29 \pm 2.45$ & -6.80 & $0.000^{*}$ \\
L4-L5 angle & $10.01 \pm 3.28$ & $13.35 \pm 2.93$ & & \\
\hline
\end{tabular}

$* \mathrm{p}<0.05$, Mean \pm SD

Table 5. Comparison of lumbar lordosis angle according to mobilization(Unit: ${ }^{\circ}$ )

\begin{tabular}{lllll}
\hline & Pre-test & Post-test & t & p \\
\cline { 2 - 4 } & Means \pm SD & Means \pm SD & & $0.000^{*}$ \\
\hline Lordosis angle & $39.96 \pm 8.57$ & $52.52 \pm 8.06$ & -14.37 & 0 \\
\hline
\end{tabular}

$* \mathrm{p}<0.05$, Mean \pm SD 


\section{Discussion}

This study applied the PA joint mobilization technique to L3 of the Subjects with no back pain. The differences in the lumbar displacement, intervertebral height, intervertebral angle, and lumbar lordosis angle before and after joint mobilization were measured to obtain fundamental clinical data on the direct and indirect therapeutic effects of joint mobilization.

The results showed consistent patterns of lumbar segmental mobility under the application of PA mobilization. Kulig et al., [15] described the upper segmental mobility under the application of PA joint mobilization to L3, where the tension in the zygapophyseal joint capsule of L2 and L3 caused the segmental mobility of L2. It has been found that a series of indirect displacements occurs due to the L1 segmental mobility. In addition, the contact between the L3 zygapophyseal inferior articular joint and the L4 zygapophyseal superior articular joint produces an indirect displacement, which can also cause sequential segmental mobility of L5. Because the posterior joint of the spine is a live joint, the articular capsule covering the cartilage surface induces and restricts the relative displacement and rotation of the adjacent spine and resists tension [16]. The articular capsule thickness of the lumbar is $2.0 \mathrm{~mm}$ in the posterior region, the maximum thickness in the front region is $3.2 \mathrm{~mm}$, and the superior and inferior areas are $2.4 \mathrm{~mm}$ thick [17]. It comprises a bunch of dense collagen fibers linked by proteoglycans and contains elastin fibers and fibrous subcells [18]. Collagen fibers are located in different directions along the superior and inferior axes of the articular capsule ligament, and wrinkles are formed (Yamashita et al., 1996). Wrinkled collagen fibers can be used to relax the articular capsule and reach mechanical limits, causing displacement and rotation of the lumbar without causing local damage [16]. Therefore, in this study, joint mobilization was applied to L3, and spinal displacement was observed most frequently from $24.51 \pm 2.25$ to $27.73 \pm 2.10 \mathrm{~cm}$ in direct lumbar L3. The displacement in L2, which is adjacent to $\mathrm{L} 3$, was from $23.81 \pm 2.30 \mathrm{~cm}$ to $26.84 \pm$ $2.14 \mathrm{~cm}$, and that in L4 was from $24.77 \pm 2.08 \mathrm{~cm}$ to $27.82 \pm 1.98 \mathrm{~cm}$, followed by more displacement. The smallest displacement was observed in L1 (from 23.09 $\pm 2.36 \mathrm{~cm}$ to $25.71 \pm 2.15 \mathrm{~cm}$ ) and L5 (from $24.35 \pm$ $1.73 \mathrm{~cm}$ to $26.93 \pm 1.80 \mathrm{~cm}$ ); however, the difference was statistically significant. No displacement was observed between the vertebrae segments, which is the movement of each vertebrae, and this chain displacement of the adjacent vertebrae increased the overall lumbar angle from $39.96 \pm 8.57^{\circ}$ to $52.52 \pm 8.06^{\circ}$.

Meanwhile, Kulig et al., [15] showed a difference of $2-4^{\circ}$ in the superior segments L1 and L2 when measuring the intervertebral angle under the application of joint mobilization to L3. The more inferior the segment, the smaller was the change in the angle $\left(1-2^{\circ}\right)$. However, in this study, the intervertebral height was measured from the changes in spinal segmentation and lumbar lordosis angle. In the dorsal vertebrae, no difference in the intervertebral height was observed in the superior segment of L3, while an increase in the height was observed in the inferior segment. In the ventral vertebrae, the intervertebral height increased for all segments. With the increase in the intervertebral height, the intervertebral angle increased from $6.21 \pm$ $2.27^{\circ}$ to $8.38 \pm 1.97^{\circ}$. Similarly, the angle for L2-L3 increased from $6.64 \pm 1.77^{\circ}$ to $9.86 \pm 1.81^{\circ}$, that for L3 $-\mathrm{L} 4$ increased from $8.23 \pm 2.23^{\circ}$ to $11.29 \pm 2.45^{\circ}$, and that for L4-L5 increased from $10.01 \pm 3.28^{\circ}$ to $13.35 \pm$ $2.93^{\circ}$. Thus, the more inferior the segment, the greater was the change in the intervertebral angle. This change, along with that in the lumbar lordosis angle, increased the intervertebral angle of all lumbar vertebrae, leading to rotation on the sagittal plane of the lumbar and, thus, extension of the entire lumbar.

The joint mobilization of the spine can control the pain and dysfunction by activating the nerve distribution tissue located in the cartilage and synovial membrane as well as the articular capsule of the zygapophyseal joint of the lumbar [19]. Godges et al., [6] indicated that lumbar mobilization stimulates the nerve cells by promoting the normal movement of damaged joints and facilitating nutrition, thus improving the functional disorders caused by pain and muscle imbalance. As these joint mobilizations have achieved therapeutic benefits in several prior studies, segmental mobility resulting from lumbar mobilization is considered to play an important role in inducing therapeutic effects.

Therefore, the lumbar strength based on the segmental mobility determined in this study can be used to 
evaluate the lumbar strength. In addition, the functional movements can be improved by applying direct or indirect PA joint mobilization.

\section{Conclusions}

The results of this study confirmed that, under the application of PA joint mobilization to L3, the kinetic movement of the lumbar produces segmental mobility. This results in direct segmentation of the joint mobilization, as well as the movement of adjacent segments, yielding sequential segmental mobility. Based on these findings, we suggest that the segmental mobility achieved through indirect approaches plays an important role in inducing therapeutic effects in patients with back pain.

\section{Conflicts of Interest}

The authors declare no conflicts of interest.

\section{References}

1. Gross AR, Kay T, Hondras M, Goldsmith C, Haines T, Peloso P, Kennedy C, Hoving J. Manual therapy for mechanical neck disorders: a systemic review. Manual Therapy. 2002;7(3);131-149.

2. Reuter U, Häling M, Kavuk I, Einhäpl, Schielke E. Vertebral artery dissections after chiropractic neck manipulation in Germany over three years. Journal Neurology. 2006;253(6);724-730.

3. Bolton PS. Spinal manipualation and spinal mobilization influence different axial sensory beds. Medical Hypotheses. 2006;66(2);258-62.

4. Twomey LT. A rationale for the treatment of back pain and joint pain by manual therapy. Phsycal Therapy. 1992;72(12);885-892.

5. Loew M, Heichel TO, Lehner B. Intararticular lesions in primary frozen shoulder after manipulation under general anesthesia. Journal of Shoulder and Elbow Surgery. 2005;14(1);16-21.

6. Godges JJ, Mattson-Bell M, Thorpe D, et al. The immediate effects of soft tissue mobilization with proprioceptive neuromuscular facilitation on glenohumeral external rotation and overhead reach. Journal of Orthopaedic \& Sports Physical Therapy. 2003;33(12);713-718.
7. Maitland, GD., Hengeveld, E., Banks, K., \& English, K. Maitland's vertebral manipulation. 7th ed. Elsevier Health Sciences. Oxford: Butterworth-Heinemann; 2005.

8. Gerti, B-D., \& Renate, W. IMTA course handbook; Level 1 foundation of the manual therapy. IMTA Business Centre. 2018.

9. Threlkeld AJ. The effects of manual therapy on connective tissue. Physical Therapy. 1992;72;893-902.

10. Petty NJ, Maher C, Latimer J, Lee M. Manual examination of accessory movements -seeking R1. Manual Therapy. 2002;7;39-43.

11. Maitland GD. Vertebral manipulation. 5th ed. Health Sciences. Oxford: Butterworth-Heinemann. 1986.

12. Gross, J. M., Fetto, J., Rosen, E. Musculoskeletal Examination. John Wiley \& Sons. 2015.

13. Snodgrass, S. J., Rivett, D. A., \& Robertson, V. J. Manual forces applied during posterior-to-anterior spinal mobilization: a review of the evidence. Journal of manipulative and physiological therapeutics. 2006;29(4);316-329.

14. Lee RYW, Evans, JH. Load-displacement-time characteristics of the spine under posteroanterior mobilisation Australian Journal of Physiotherapy. 1992;38;115-123.

15. Kulig, K., Landel, R. F., \& Powers, C. M. Assessment of lumbar spine kinematics using dynamic MRI: a proposed mechanism of sagittal plane motion induced by manual posterior-to-anterior mobilization. Journal of Orthopaedic \& Sports Physical Therapy. 2004;34(2);57-64.

16. Jaumard, N. V., Welch, W. C., \& Winkelstein, B. A. Spinal facet joint biomechanics and mechanotransduction in normal, injury and degenerative conditions of biomechanical engineering. 133(7);071010.

17. Sato, S. I., Oguma, H., Murakami, G., \& Noriyasu, S. Morphometrical study of the joint surface and capsule of the lumbar zygapophysial joint with special reference to their laterality. folia anatomica Japonica. 79(1); 43-53.

18. Yamashita, T., Minaki, Y., Öaktay, A. C., Cavanaugh, J. M., \& King, A. I. A morphological study of the fibrous capsule of the human lumbar facet joint 21(5);538-543.

19. Kalichman, L., \& Hunter, D. J. Lumbar facet joint osteoarthritis: a review. Seminars in arthritis and rheumatism. 37(2);69-80. 